\title{
Migration of XEN45 Implant: Findings, Mechanism, and Management
}

\author{
Zaria C Ali ${ }^{1}$, Dawnn I Khoo ${ }^{2}$, Francessco Stringa ${ }^{3}$, Vikas Shankar ${ }^{4}$
}

\begin{abstract}
Aim: The aim of this study is to report the mechanism of XEN migration and its management.

Background: Over the past decade, new less invasive surgical approaches for glaucoma have been devised and carried out successfully. One such technique is the use of the XEN gel stent. We present a rare and relatively unknown complication of XEN migration and present in detail the likely mechanism by which this occurs, and its subsequent management.

Case description: A 73-year-old male with primary angle closure on maximal medical treatment presented with an intraocular pressure of $30 \mathrm{~mm} \mathrm{Hg}$ in the left eye. The visual acuity was $6 / 5$, iridocorneal angles were open in all four quadrants, and the cup disc ratio was 0.4 . As phacoemulsification alone was unlikely to adequately lower intraocular pressures, the patient underwent combined phacoemulsification and XEN implantation. Although the patient had a good postoperative result with pressures lowered to $11 \mathrm{~mm} \mathrm{Hg}, 4$ months after the operation, the XEN was found to have migrated $4 \mathrm{~mm}$ into the anterior chamber, associated with a low-grade uveitis. The patient subsequently had the XEN explanted a new XEN inserted. Pressures lowered 1 month postoperatively to $14 \mathrm{~mm} \mathrm{Hg}$.

Conclusion: XEN migration is likely due to a combination of mechanical and frictional forces. If the XEN is positioned such that more than $2 \mathrm{~mm}$ is in the subconjunctival space, the XEN is likely to be angled upward and, therefore, be more susceptible to these forces and undergo migration. Clinical significance: It is essential that XEN implants are correctly sited and that this is confirmed intraoperatively to prevent the need for further procedures.
\end{abstract}

Keywords: Complications, Minimally invasive procedure, Surgical treatment, XEN.

Journal of Current Glaucoma Practice (2019): 10.5005/jp-journals-10078-1253

\section{BACKGROUND}

Glaucoma is a chronic optic neuropathy which can be managed with medications, laser, or surgery. For many years, trabeculectomy was the only surgical option in these patients. However, over the past decade, new surgical approaches which are less invasive have been devised and carried out successfully. One such technique is the use of the XEN gel stent (Allergan PLC, Irvine, CA, USA) which allows subconjunctival filtration. The implant is found to have a good safety profile with very few complications. ${ }^{1,2}$ We present a rare and relatively unknown complication of XEN migration and present in detail the likely mechanism by which this occurs, and its subsequent management.

\section{Case Description}

A 73-year-old male with primary angle closure with pre-perimetric changes on optical coherence tomography (OCT) nerve fiber layer in the left eye was on maximal medical treatment including xalacom o.d., pilocarpine $2 \%$ q.d.s., simbrinza b.d., and oral acetazolamide $250 \mathrm{mg}$ SR b.d. with an IOP of $30 \mathrm{~mm} \mathrm{Hg}$. He had previously bilateral peripheral iridotomies.

Ocular examination showed a visual acuity of $6 / 5$ with patent iridotomies with gonioscopy showing that angles were open in all four quadrants with minimal peripheral anterior synichaie affecting less than $90^{\circ}$ inferiorly. A central corneal thickness was $523 \mu \mathrm{m}$ and the cup disc ratio was 0.4 . The axial length was $21.86 \mathrm{~mm}$. In view of the open angles, it was unlikely phacoemulsification on its own would achieve adequate intraocular pressure control. Therefore, we discussed risks and benefits and he underwent phacoemulsification and intraocular lens implantation combined with XEN implantation with mitomoycin C. Two months postoperatively, the IOP was
${ }^{1}$ Department of Medical Retina, Manchester Royal Eye Hospital, Manchester, UK

${ }^{2-4}$ Department of Ophthalmology, East Lancashire Teaching Hospitals, Blackburn, UK

Corresponding Author: Zaria C Ali, Department of Medical Retina, Manchester Royal Eye Hospital, Manchester, UK, Phone: +44 7715233383, e-mail: zariaali@gmail.com

How to cite this article: Ali ZC, Khoo DI, et al. Migration of XEN45 Implant: Findings, Mechanism, and Management. J Curr Glaucoma Pract 2019;13(2):79-81.

Source of support: Nil

Conflict of interest: None

$11 \mathrm{~mm} \mathrm{Hg}$ with a good position of the XEN. Four months postop, his IOP remained stable at $11 \mathrm{~mm} \mathrm{Hg}$ but the XEN implant showed signs of migration and $4 \mathrm{~mm}$ of the implant was visible in the anterior chamber (Fig. 1A). Bleb was localized around the tip of the XEN implant with conjunctiva showing no injection (Fig. 1B). The patient was also noted to have a minimal flare and increased posterior synechiae suggestive of a low-grade uveitis in the left eye. Although IOP was controlled, surgery to reposition the XEN and revise the bleb was discussed in view to prevent further inflammation and possible further XEN migration. He underwent surgery; the fornixbased conjunctival flap was made from 10 to 12 o'clock. The tip of the XEN implant was seen at the scleral bed. While explanting the old implant the tip of the XEN broke (Fig. 1C). A corneal incision was made in the opposite quadrant and the old XEN was explanted using a 23-gauge Grieshaber advanced DSP tip serrated edge through this incision. A new XEN implant was then advanced through this 


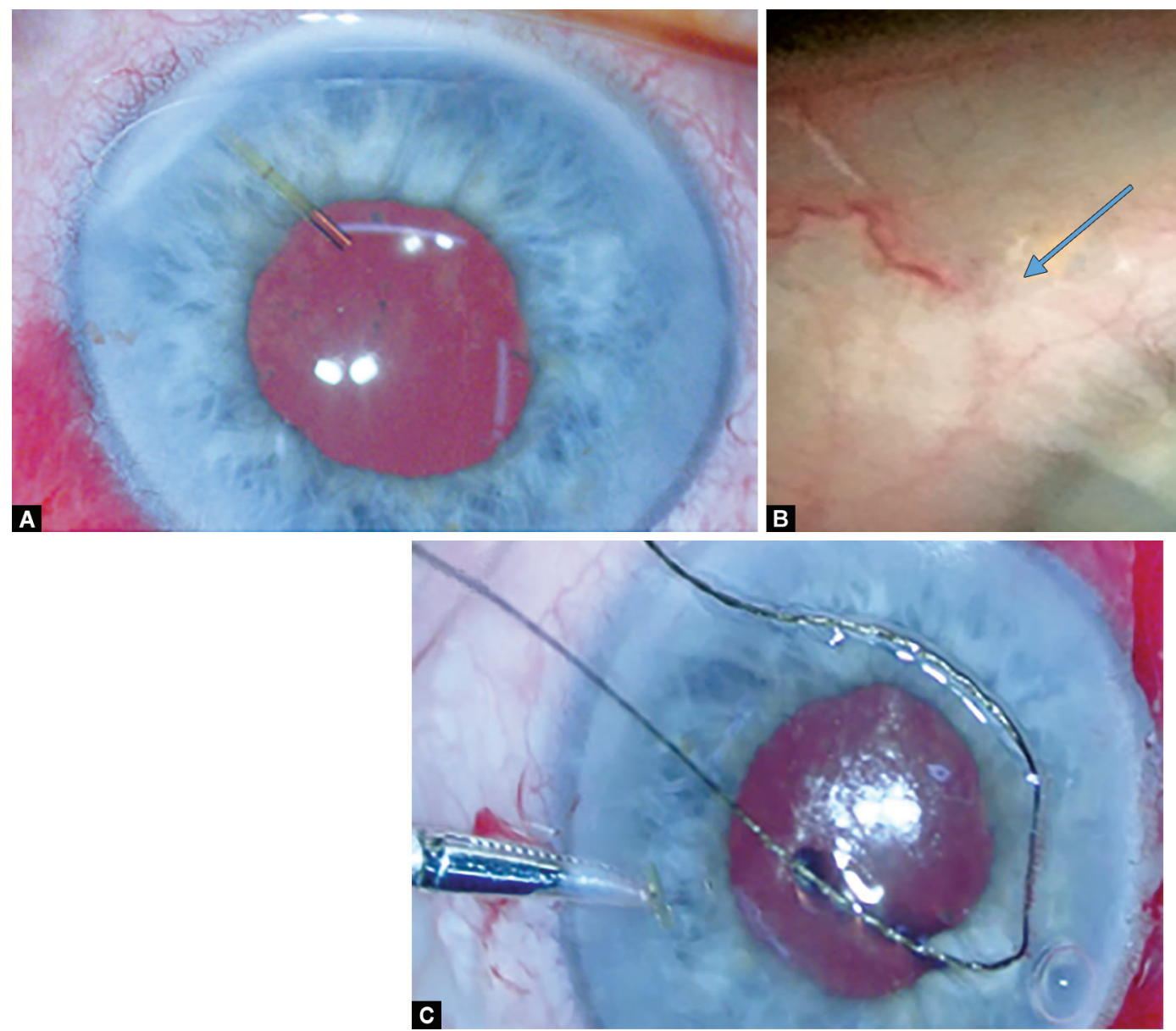

Figs $1 \mathrm{~A}$ to $\mathrm{C}$ : XEN position preoperatively and findings intraoperatively; (A) XEN migration forward, causing $4 \mathrm{~mm}$ of the implant to be visible in the anterior chamber; (B) Conjunctival bleb from the XEN tip showing the XEN tip (blue arrow); (C) The broken tip after attempted repositioning can be seen

incision into the 2o'clock position where the original XEN had been sited. On gonioscopy, the XEN was confirmed to be in the correct position. The conjunctiva was closed using three $10^{\prime} 0$ nylon sutures at the limbus. Subconjunctival mitomycin C $0.1 \mathrm{cc}$ of $0.2 \mathrm{mg} / \mathrm{mL}$ was used to prevent further inflammation and scarring. Postoperatively, the pressure in the eye was $8 \mathrm{~mm} \mathrm{Hg}$ and remained stable at 14 $\mathrm{mm} \mathrm{Hg} 2$ weeks later. One month postoperatively the pressure remained low at $16 \mathrm{~mm} \mathrm{Hg}$ on topical steroids q.d.s. and the XEN implant maintained a good position (Fig. 2).

\section{Discussion}

We present a rare complication of XEN implantation; migration of the implant. It is recommended that the XEN implant should have approximately $1 \mathrm{~mm}$ visible in the anterior chamber, exit from the sclera should be approximately $3 \mathrm{~mm}$ from the limbus, and $2 \mathrm{~mm}$ should be situated within the subconjunctival space. ${ }^{3}$ This is to prevent erosion and ensure that the XEN does not migrate. If the length of the XEN is placed more in the anterior chamber, and a smaller portion within the subconjunctival space, the implant may be more obliquely angled (Fig. 3). This would then mean that the implant is more likely to be affected by pressures exerted by the lid on blinking. This lid is known to exert a force on the globe and can cause the globe to move posteriorly by up to $6 \mathrm{~mm}$ during the closing phase of the blink. ${ }^{4}$ It has been suggested that the

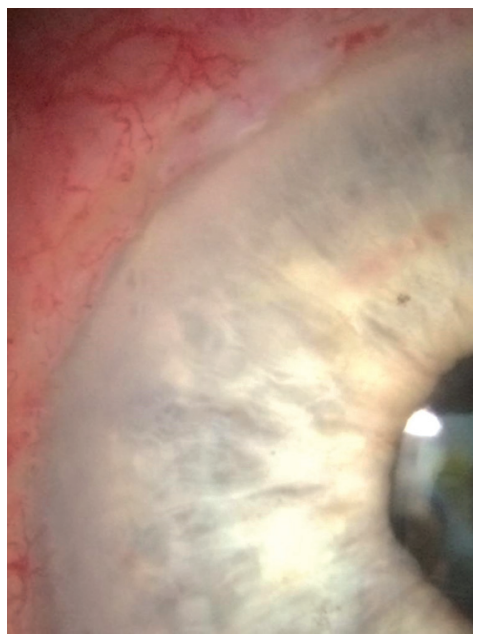

Fig. 2: Result of second surgery 4 weeks postoperatively; a good diffuse bleb is seen and the XEN has maintained a good position

orbicularis would, therefore, have sufficient power to move the bulbar conjunctiva anteriorly, and there is little counteraction when the lid is opened, meaning an overall anterior displacement. ${ }^{5}$ There could even be an additional displacement force from the lower lid which can move the conjunctiva nasally. ${ }^{5}$ With an improperly 


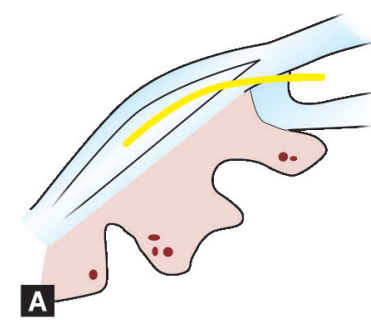

B

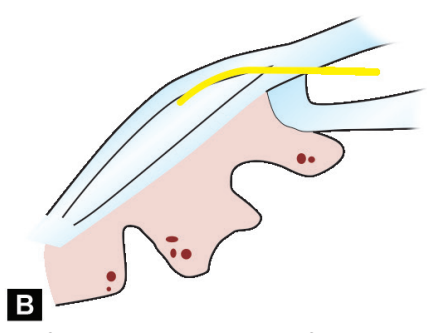

Figs $3 A$ and $B$ : How the position of the XEN may be affected. The yellow represents the XEN implant; (A) The XEN when placed $1 \mathrm{~mm}$ within the anterior chamber, $2 \mathrm{~mm}$ within the sclera and $3 \mathrm{~mm}$ within the subconjunctival space forms a well-shaped bleb, and has a stable position; (B) When the XEN is placed more anteriorly the XEN is angled obliquely and is more likely to be subject to forces from blinking, friction and microtrauma

implanted XEN, the implant is more likely to be affected by these forces.

It has also been postulated that further frictional forces can lead to microtrauma which may be significant enough to cause pathology in the conjunctiva such as conjunctival folds or superior limbic keratoconjunctivitis. ${ }^{5,6}$ Frictional forces may be generated if there is disruption of the tear film, surface quality, difference in load between different layers within the eye, and altered lid dynamics. ${ }^{5,6}$ Ocular surface disease is common in patients with glaucoma and may be present in up to $59 \%$ of patients. ${ }^{7}$ These patients would, therefore, have an altered tear film and poor ocular surface quality which could lead to increased frictional forces and micro-trauma which could affect the bleb, and contribute to the movement of the XEN.

The XEN itself has many properties which are help prevent migration and erosion. ${ }^{8}$ This includes its ability to become flexible once implanted in the eye, allowing it to conform to the tissue shape, but also the adoption of an " $\mathrm{S}$ " curve as it goes through the scleral channel and bends under the conjunctiva. ${ }^{8}$ The smaller the lumen of the XEN, the greater the flexibility. ${ }^{8}$ If there were any issues with the flexibility of the XEN it may have meant it was less able to conform the tissue shape, and may not have adopted the " $S$ " curve which helps prevent migration. This coupled with frictional and mechanical forces from the lid may have caused migration of the XEN.

\section{ConCLUSION}

We feel that XEN migration is likely multifactorial on the basis of the presence of ocular surface disease causing an increase in both mechanical and frictional forces leading to migration of the XEN, and properties of the individual XEN itself. There is only one other report of XEN migration which occurred 2 months after needling revision and there were subsequent endothelial cell loss and a corneal opacity. ${ }^{9}$ This is somewhat similar to one case of complete XEN dislocation which occurred after attempted manipulation of the XEN. ${ }^{10}$ Unlike these cases where there was movement of the XEN after a secondary procedure, the XEN in our case migrated after seemingly uncomplicated surgery and did not cause a rise in intraocular pressure.

\section{Clinical Significance}

Our case report, therefore, emphasizes the importance of the physics of the forces that can act on the XEN implant if it is not properly placed. If less than $2 \mathrm{~mm}$ is placed within the subconjunctival space the XEN can be angled upwards and will, therefore, be more susceptible to the mechanical and frictional forces causing it to move toward the anterior chamber. IOP may remain controlled even with movement of the XEN so careful examination of the position of the implant is imperative to ensure that any movement is picked up early allowing appropriate follow-up and management.

\section{References}

1. De Gregorio A, Pedrotti E, et al. XEN glaucoma treatment system in the management of refractory glaucomas: a short review on trial data and potential role in clinical practice. Clin Ophthalmol 2018;12:773-782. DOI: 10.2147/OPTH.S146919.

2. Grover DS, Flynn WJ, et al. Performance and Safety of a New Ab Interno Gelatin Stent in Refractory Glaucoma at 12 Months. Am J Ophthalmol 2017;183:25-36. DOI: 10.1016/j.ajo.2017.07.023.

3. VAIVera, Stalmans I, et al. Gel stent implantation-recommendations for preoperative assessment, surgical tecnique and postoperative managment. US Ophthalmic Review 2018;11(1):38-46. DOI: 10.17925/ USOR.2018.11.1.38.

4. Doane MG. Interactions of eyelids and tears in corneal wetting and the dynamics of the normal human eyeblink. Am J Ophthalmol 1980;89(4):507-516. DOI: 10.1016/0002-9394(80)90058-6.

5. Cher I. Blink-related microtrauma: when the ocular surface harms itself. Clin Exp Ophthalmol 2003;31(3):183-190.

6. Pult $\mathrm{H}$, Riede-Pult BH, et al. The Relation Between Blinking and Conjunctival Folds and Dry Eye Symptoms. Optom Vis Sci 2013;90(10):1034-1039. DOI: 10.1097/OPX.0000000000000029.

7. Leung EW, Medeiros FA, et al. Prevalence of ocular surface disease in glaucoma patients. J Glaucoma 2008;17(5):350-355. DOI: 10.1097/ IJG.0b013e31815c5f4f.

8. Lewis RA. Ab interno approach to the subconjunctival space using a collagen glaucoma stent. J Cataract Refract Surg 2014;40(8): 1301-1306. DOI: 10.1016/j.jcrs.2014.01.032.

9. Gillmann K, Bravetti GE, et al. Anterior Chamber XEN Gel Stent Movements: The Impact on Corneal Endothelial Cell Density. J Glaucoma 2019 Jun;28(6):e93-e95. DOI: 10.1097/ IJG.0000000000001200.

10. Atalay Kursat NSA. Kirgiz Ahmet. Complication of micro-bypass stenting: the anterior chamber displacement of a Xen implant. Arq Bras Oftalmol 2018;81(6):514-516. DOI: 10.5935/0004-2749.20180098. 